\title{
TAZ as a novel regulator of oxidative damage in decidualization via Nrf2/ARE/Foxo1 pathway
}

\author{
Hai-Fan Yu $\mathbb{D}^{1,3}$, Lian-Wen Zheng ${ }^{2,3}$, Zhan-Qing Yang ${ }^{1}$, Yu-Si Wang ${ }^{1}$, Ting-Ting Wang ${ }^{1}$, Zhan-Peng Yue ${ }^{1 凶}$ and Bin Guo ${ }^{1 \times}$
}

(c) The Author(s) 2021

TAZ, as a crucial effector of Hippo pathway, is required for spermatogenesis and fertilization, but little is known regarding its physiological function in uterine decidualization. In this study, we showed that TAZ was localized in the decidua, where it promoted stromal cell proliferation followed by accelerated G1/S phase transition via Ccnd3 and Cdk4 and induced the expression or activity of stromal differentiation markers Prl8a2, Prl3c1 and ALP, indicating the importance of TAZ in decidualization. Knockdown of TAZ impeded HB-EGF induction of stromal cell proliferation and differentiation. Under oxidative stress, TAZ protected stromal differentiation against oxidative damage by reducing intracellular ROS and enhancing cellular antioxidant capacity dependent on the Nrf2/ARE/Foxo1 pathway. TAZ strengthened the transcriptional activity of Nrf2 which directly bound to the antioxidant response element (ARE) of Foxo1 promoter region. Additionally, silencing TAZ caused accumulation of intracellular ROS through heightening NOX activity whose blockade by APO reversed the disruption in stromal differentiation. Further analysis revealed that TAZ might restore mitochondrial function, as indicated by the increase in ATP level, mtDNA copy number and mitochondrial membrane potential with the reduction in mitochondrial superoxide. Additionally, TAZ modulated the activities of mitochondrial respiratory chain complexes I and III whose suppression by ROT and AA resulted in the inability of TAZ to defend against oxidative damage to stromal differentiation. Moreover, TAZ prevented stromal cell apoptosis by upregulating Bcl2 expression and inhibiting Casp3 activity and Bax expression. In summary, TAZ might mediate HB-EGF function in uterine decidualization through Ccnd3 and ameliorate oxidative damage to stromal cell differentiation via Nrf2/ARE/Foxo1 pathway.

Experimental \& Molecular Medicine (2021) 53:1307-1318; https://doi.org/10.1038/s12276-021-00655-2

\section{INTRODUCTION}

In response to implanting embryos, uterine stromal cells proliferate and then differentiate into decidual cells, a process known as decidualization, which is required for pregnancy maintenance $^{1,2}$. Defective decidualization can lead to a range of pregnancy disorders, including embryo miscarriage, recurrent spontaneous abortion, and early pregnancy loss ${ }^{2,3}$. Although numerous genes and signaling pathways have been demonstrated to potentially participate in the process of decidualization, the underlying regulatory mechanisms remain largely unknown.

Transcriptional coactivator with PDZ-binding motif (TAZ), also referred to as WW domain-containing transcription regulator 1 (Wwtr1), is a downstream effector of Hippo pathway that has been established as a principal modulator of organ size, cancer development and stem cell fate, as well as cell proliferation and differentiation ${ }^{4-6}$. Deletion of TAZ in mice reduced litter size and accelerated the lethality of Yes-associated protein (YAP)-deficient embryos $^{7,8}$. In addition, TAZ was detected in spermatogenic and interstitial cells, where its deficiency led to abnormalities in testicular structure and function along with diminished sperm counts and fertility ${ }^{9}$. In zebrafish, TAZ was indispensable for fertilization ${ }^{10,11}$. Although abundant TAZ protein has been found in the nuclei of human endometrial stromal cells ${ }^{12}$, its physiological significance in decidualization has not been reported.
Oxidative stress (OS) is characterized by the disequilibrium between reactive oxygen species (ROS) generation and antioxidant defense and is involved in the pathogenesis of female infertility ${ }^{13,14}$. Accumulating evidence has demonstrated that OS decreases the number of embryos implanted and impairs uterine decidualization, and it is associated with a variety of reproductive disorders, such as endometriosis, recurrent pregnancy loss, and preeclampsia ${ }^{13-15}$. In the context of OS, elevated TAZ protein was transiently observed in HEK $293 \mathrm{~T}$ cells $^{16}$, implying that TAZ might function as a redox sensor. However, there is limited information regarding whether TAZ ameliorates oxidative damage to decidualization.

The present study suggested the importance of TAZ in uterine decidualization through $\mathrm{Ccnd} 3$ and $\mathrm{Cdk} 4$ in response to HB-EGF. Furthermore, TAZ might protect stromal differentiation from oxidative damage by restoring cellular antioxidant capacity dependent on the Nrf2/ARE/Foxo1 pathway and preventing mitochondrial dysfunction and cell apoptosis under OS.

\section{MATERIALS AND METHODS}

\section{Uterine tissue collection}

Mouse uteri from days $1-8$ of pregnancy and undergoing artificial decidualization were gathered as described previously ${ }^{17}$. All animal

${ }^{1}$ College of Veterinary Medicine, Jilin University, Changchun, PR China. ${ }^{2}$ Reproductive Medical Center, the Second Hospital of Jilin University, Changchun, PR China. ${ }^{3}$ These authors contributed equally: Hai-Fan Yu, Lian-Wen Zheng. ${ }^{\circledR}$ email: yuezp@jlu.edu.cn; guobin79@jlu.edu.cn

Received: 5 January 2021 Revised: 24 May 2021 Accepted: 15 June 2021

Published online: 8 September 2021 
experimental procedures were approved by the Committee for the Ethics on Animal Care and Use of Jilin University (SY201905031).

\section{In situ hybridization}

In situ hybridization was performed as depicted previously ${ }^{17}$. Briefly, a TAZ CRNA probe was labeled with digoxigenin and then used for hybridization in frozen sections. After incubation with sheep anti-digoxigenin antibody conjugated to alkaline phosphatase, sections were visualized with BCIP/ NBT followed by counterstaining with methyl green. The TAZ primers used for in situ hybridization were listed in Supplementary Table 1.

\section{In vitro decidualization}

In vitro decidualization was performed by replenishing estradiol-17 $\beta$ (10 $\mathrm{nM}$, Sigma) plus progesterone $(1 \mathrm{mM}$, Sigma) in uterine stromal cells obtained on the 4th day of pregnancy as depicted previously ${ }^{17}$.

\section{Immunofluorescence}

Decidual cells obtained on day 7 of pregnancy were seeded on glass coverslips to perform immunofluorescence as depicted previously ${ }^{17}$. Briefly, after fixation with $4 \%$ cold paraformaldehyde, the cells were incubated overnight with antibody against TAZ (1:100, Thermo Fisher Scientific) followed by the addition of goat anti-rabbit Alex Fluor 488 conjugated antibody (1:1000, Invitrogen) and DAPI counterstaining of the nuclei. Images were obtained by confocal microscopy.

\section{Western blotting}

Proteins from uteri or stromal cells were separated by SDS-PAGE and then transferred onto PVDF membranes. After blocking with 5\% skim milk, the membranes were probed overnight with antibodies against TAZ (1:1000, Cell Signaling Technology), phospho-TAZ (Ser89, 1:1000, Cell Signaling Technology), phospho-large tumor suppressor kinase (phospho-LATS, Thr1079, 1:1000, Cell Signaling Technology), nuclear factor erythroid 2-related factor 2 (Nrf2, 1:1000, Proteintech), forkhead box 01 (Foxo1, 1:1000, Proteintech) or Gapdh (1:5000, Proteintech) at $4{ }^{\circ} \mathrm{C}$ followed by incubation with HRP-conjugated secondary antibody (1:5000). Signals were visualized with an ECL chemiluminescent kit.

\section{Real-time PCR}

The expression levels of TAZ, prolactin family 8 , subfamily a, member 2 (Prl8a2), prolactin family 3, subfamily c, member 1 (Prl3c1), cyclin A1 (Ccna1), Ccnb1, Ccnb2, Ccnd1, Ccnd3, Ccne1, cyclin-dependent kinase 1 (Cdk1), Cdk2, Cdk4, Cdk6, caspase 3 (Casp3), B cell leukemia/lymphoma 2 (Bcl2), Bcl2associated $X$ protein (Bax), Nrf2 and Foxo1, as well as mitochondrial DNA (mtDNA) copy number, were determined by real-time PCR analysis using a Roche LightCycler 96 Detection System as described previously ${ }^{15}$. The primers used for TAZ, Nrf2 and Foxo1 were listed in Supplementary Table 1, and the other primers were previously described ${ }^{15,18}$.

\section{Plasmid construction and transfection}

Plasmids overexpressing TAZ variant TAZa and TAZb were constructed as described previously ${ }^{17}$, and the primer sequences were provided in Supplementary Table 1. Transfection of this overexpression plasmid was performed with Lipofectamine 3000 according to the manufacturer's protocol. After introduction of TAZ overexpression plasmid, stromal cells were collected at 12,24 , and $48 \mathrm{~h}$ in the absence or presence of estradiol$17 \beta$ plus progesterone. For further analysis, cells were transfected with TAZ overexpression plasmid and then exposed to $100 \mu \mathrm{M} \mathrm{H}_{2} \mathrm{O}_{2}$ in the absence or presence of superoxide dismutase (SOD) inhibitor diethyldithiocarbamate (DDC, $10 \mu \mathrm{M}$, Sigma), catalase (CAT) inhibitor 3-amino-1:2:4-triazole (ATZ, $1 \mathrm{mM}$, Sigma), glutathione peroxidase (GPX) inhibitor mercaptosuccinic acid (MS, $300 \mu \mathrm{M}$, Sigma), glutathione reductase (GR) inhibitor 1,3-bis (2-chloroethyl)-1-nitrosourea (BCNU, $20 \mu \mathrm{M}$, Sigma), GSH synthesis inhibitor buthionine sulfoximine (BSO, $10 \mu \mathrm{M}$, Sigma), mitochondrial respiratory chain complex I inhibitor rotenone (ROT, $2 \mu \mathrm{M}, \mathrm{MCE}$ ), mitochondrial respiratory chain complex III inhibitor antimycin A (AA, $2 \mu \mathrm{M}, \mathrm{Abcam}), \mathrm{Nrf2}$ inhibitor ML385 (10 $\mu \mathrm{M}, \mathrm{MCE})$ or Foxo1 inhibitor AS1842856 (1 $\mu \mathrm{M}, \mathrm{MCE})$ under in vitro decidualization.

\section{RNA interference}

The following small-interfering RNAs (siRNAs) targeting TAZ were synthesized by GenePharma: CCCUCUUCAACUCUGUCAUTT (siRNA 1),
CCACUGGCCAGAGAUACUUTT (siRNA 2), and GCUCAGAUCCUUUCCUCA ATT (siRNA 3). Control siRNA (negative control) sequence was described previously ${ }^{17}$. After introduction of TAZ siRNA into stromal cells using the aforementioned Lipofectamine 3000 protocol, the cells were harvested at 12,24 , and $48 \mathrm{~h}$ in the absence or presence of estradiol-17 $\beta$ plus progesterone. For further analysis, cells were treated with TAZ siRNA followed by the addition of recombinant HB-EGF protein (rHB-EGF, $100 \mathrm{ng} /$ $\mathrm{ml}$, R\&D Systems). In addition, TAZ siRNA-transfected stromal cells were exposed to $100 \mu \mathrm{M} \mathrm{H}_{2} \mathrm{O}_{2}$ for $4 \mathrm{~h}$ with/without the NADPH oxidase (NOX) inhibitor apocynin (APO, $1 \mu \mathrm{M}, \mathrm{MCE})$ under in vitro decidualization.

\section{Cell proliferation}

Twenty-four hours after introduction of TAZ overexpression plasmid or siRNA into stromal cells in the absence or presence of rHB-EGF, cells were supplemented with MTS reagent (Promega) and incubated for 2-4 h. The absorbance was measured with multimode reader.

\section{Cell cycle analysis}

After introduction of TAZ overexpression plasmid or siRNA into stromal cells for different time courses in the absence or presence of rHB-EGF, cells were fixed, stained with $\mathrm{PI} / \mathrm{RNase}$ staining buffer (BD Biosciences) and then analyzed by flow cytometry as described previously ${ }^{15}$.

\section{Alkaline phosphatase (ALP) activity assay}

After different treatments, stromal cells were lysed, and ALP activity was measured in accordance with the corresponding assay kit (Beyotime).

\section{Determination of ROS level}

After transfection with TAZ siRNA or overexpression plasmid and then exposure to $\mathrm{H}_{2} \mathrm{O}_{2}$ in the absence or presence of ML385 or AS1842856 during in vitro decidualization, cells were incubated with the fluorescent probe DCFH-DA (Beyotime, $20 \mu \mathrm{M}$ ), dihydroethidium (Beyotime, $10 \mu \mathrm{M}$ ), or MitoSOX Red mitochondrial superoxide indicator (Invitrogen, $5 \mu \mathrm{M}$ ) at $37^{\circ} \mathrm{C}$ for different times. The cells were analyzed with multidetection microplate reader or flow cytometry to determine the levels of intracellular $\mathrm{ROS}, \mathrm{O}_{2}{ }^{-}$and mitochondrial $\mathrm{O}_{2}{ }^{-}$.

\section{Measurement of pro- and anti-oxidant parameters}

After treatment, proteins were extracted to assess the contents of malondialdehyde (MDA) and reduced glutathione (GSH) as well as GSH/ oxidized glutathione (GSSG) ratio and to determine the activities of SOD, CAT, GPX, GR and NOX in accordance with the corresponding assay kit (Beyotime or Solarbio).

\section{Measurement of mitochondrial membrane potential and ATP content}

After treatment, stromal cells were incubated with the JC-1 fluorescent probe and then analyzed by flow cytometry using a mitochondrial membrane potential assay kit (Beyotime). In addition, ATP content was calculated by the corresponding assay kit (Beyotime).

\section{Assessment of mitochondrial respiratory chain complex I, II and III activities}

After transfection with TAZ siRNA or overexpression plasmid and then exposure to $\mathrm{H}_{2} \mathrm{O}_{2}$ under in vitro decidualization, the activities of mitochondrial respiratory chain complexes I, II and III were calculated by the corresponding assay kit (Solarbio).

\section{Cell apoptosis analysis}

After treatment, cell apoptosis was evaluated by flow cytometry with an Annexin V-FITC apoptosis detection kit according to the manufacturer's instructions (Beyotime). In addition, Casp3 activity was determined with the corresponding assay kit (Beyotime).

\section{Dual-luciferase reporter gene assay}

After Foxo1 promoter sequences $(+217$ to +416$)$ containing the Nrf2/AREbinding site were inserted into $\mathrm{PGL} 4.18$ vector and then cotransfected with TAZ overexpression plasmid with/without Nrf2 inhibitor ML385, luciferase activity was measured with dual-luciferase reporter gene assay kit (Beyotime). In addition, pARE-luc (Beyotime) or 8xGTIIC-luciferase plasmid 
(Addgene) was introduced into stromal cells to assess Nrf2 or TAZ-TEAD (TEA domain) transcriptional activity. The pRL-SV40 plasmid (Beyotime) was used for data normalization.

\section{Statistical analysis}

All data were analyzed with the SPSS19.0 software program. Significant difference between two groups was compared with independent-samples $t$ tests. Multiple comparisons were evaluated with one-way ANOVA. All values were shown as the means \pm SEM. $P<0.05$ was considered statistically significant.

\section{RESULTS}

Location and expression of TAZ in pregnant uteri

To investigate the relevance between TAZ and early pregnancy events, in situ hybridization was applied to localize TAZ mRNA in pregnant uteri. The results showed that TAZ mRNA was distributed in luminal and glandular epithelia from days 1 to 3 of pregnancy, and exhibited a weak signal in the uteri at day 4 of pregnancy (Fig. 1a). With the onset of implantation, TAZ mRNA was dramatically increased in the subluminal stroma surrounding the implanting blastocyst, but no visible signal was detected at the interimplantation site (Fig. 1a). From days 6 to 8 of pregnancy, uterine decidua showed an apparent signal for TAZ mRNA (Fig. 1a). Real-time PCR and Western blotting analyses verified abundant TAZ mRNA and total protein accumulation in uteri on days 6-8 of pregnancy, but phosphorylated TAZ level was obviously reduced (Fig. 1 b, Supplementary Fig. 1a). Immunocytochemistry showed marked nuclear localization of TAZ protein in decidual cells (Fig. 1c). In addition, the mRNA and protein levels of TAZ in decidual cells were higher than those in nondecidualized stromal cells, but TAZ phosphorylation level was decreased (Supplementary Fig. 1b, c).

TAZ expression in artificially induced decidual issues and cells To confirm the potential involvement of TAZ in decidualization, we employed in vivo- and in vitro-induced decidualization models. Under oil-infused artificial decidualization, TAZ mRNA signal was mostly localized to decidualizing stromal cells but was not visible in the uninjected control uteri (Fig. 1d). Quantitative analysis of TAZ revealed elevated $m R N A$ and total protein levels in oil-treated uteri, whereas TAZ phosphorylation level remained steady (Fig. 1e, Supplementary Fig. 1d). Moreover, after stromal cells were induced to decidualize, the expression of TAZ mRNA and total protein exhibited a noticeable increase for 30 and $45 \mathrm{~min}$ followed by relatively steady expression at 60 and $120 \mathrm{~min}$, whereas TAZ phosphorylation level was decreased, except at $120 \mathrm{~min}$, when it was indistinguishable from that of the control (Fig. 1f, Supplementary Fig. 1e).

\section{TAZ function in decidualization}

Considering the high expression of TAZ in decidual cells, we hypothesize that TAZ is important to uterine decidualization, where stromal cells undergo extensive proliferation and differentiation. To test this hypothesis, TAZ function in stromal cell proliferation was appraised. After introduction of TAZ overexpression plasmid, which dramatically increased the corresponding mRNA and total protein levels and TAZ-TEAD transcriptional activity along with slight elevation for TAZ phosphorylation, stromal cell proliferation rate exhibited an obvious enhancement (Fig. 2a, Supplementary Fig. 2a-c). As indicated by flow cytometry analysis, overexpression of TAZ resulted in an accelerated $\mathrm{G} 1$ to $\mathrm{S}$ phase transition at $24 \mathrm{~h}$ but not at 12 or $48 \mathrm{~h}$ (Fig. $2 \mathrm{~b}, \mathrm{c}$ ). In contrast, after introduction of TAZ siRNA 2 and 3, which effectively repressed TAZ mRNA and protein levels and weakened TAZ-TEAD transcriptional activity concomitant with notable inhibitory effects for TAZ siRNA 3, stromal cell proliferation was attenuated and G1/S phase transition was stalled at $24 \mathrm{~h}$
(Fig. 2d-f, Supplementary Fig. 2d-f). Then, the expression of key cell cycle regulators, namely, cyclins (Ccns) and cyclin-dependent kinases (Cdks), were measured. Constitutive activation of TAZ upregulated the expression of $\mathrm{Ccnd} 3$ and $\mathrm{Cdk} 4$, while silencing TAZ led to the opposite effect (Fig. $2 g, h$ ). TAZ did not seem to have a distinct impact on the mRNA levels of Ccna1, Ccnb1, Ccnb2, Ccnd1, Ccne1, Cdk1, Cdk2, or Cdk6 (data not shown).

To determine the significance of TAZ in stromal cell differentiation, we assessed its regulation of stromal cell differentiation markers Prl8a2 and Prl3c1 expression and ALP activity ${ }^{17,19}$. Sustained activation of TAZ strengthened the levels of Prl8a2 and Prl3c1 mRNA expression as well as ALP activity regardless of the existence or not of estrogen and progesterone (Fig. $2 \mathrm{i}-\mathrm{k}$ ). In contrast, knockdown of TAZ markedly repressed Prl8a2 and Prl3c1 expression and weakened ALP activity (Fig. 2I-n).

\section{TAZ mediated HB-EGF function in decidualization}

Heparin-binding EGF-like growth factor (HB-EGF) is essential for uterine decidualization ${ }^{20,21}$. Supplementation with exogenous rHBEGF facilitated the expression of TAZ mRNA and total protein concomitant with an apparent decrease in TAZ phosphorylation (Fig. 3a, b). LATS is required for phosphorylation of TAZ which is retained in the cytoplasm for proteasomal degradation ${ }^{5,22}$. Under in vitro decidualization conditions, HB-EGF evidently restrained the phosphorylation of LATS to some extent (Fig. 3b). Because of the lack of a DNA-binding domain, unphosphorylated TAZ executes its transcriptional activity by interacting with TEAD transcription factors after translocation into the nucleus ${ }^{22}$. After introduction of 8xGTIIC-luciferase plasmid which reflected TAZ-TEAD transcriptional activity, HB-EGF remarkably intensified the luciferase activity (Fig. 3c). We next wonder whether TAZ is involved in HB-EGF function in decidualization. After import of siRNA against TAZ, rHBEGF failed to induce stromal cell proliferation and elevate the proportion of S-phase cells accompanied by the reduction of Cond 3 and Cdk4 expression (Fig. 3d-h). In addition, silencing TAZ partially neutralized the mediation of HB-EGF on stromal cell differentiation, as indicated by the decline in Prl8a2 and Prl3c1 expression and ALP activity (Fig. 3i-k).

\section{TAZ protected uterine stromal cell differentiation from oxidative disruption}

Among the many factors affecting pregnancy, OS caused by excessive ROS generation has received much attention ${ }^{13,14}$. Therefore, we generated an $\mathrm{H}_{2} \mathrm{O}_{2}$-induced OS model to investigate whether TAZ was involved in regulating the redox state of stromal cells during decidualization. After cell exposure to $\mathrm{H}_{2} \mathrm{O}_{2}$, TAZ mRNA and total protein levels, as well as TAZ-TEAD transcriptional activity, were markedly increased during in vitro decidualization, whereas phosphorylation of TAZ and LATS revealed a notable obvious decline (Fig. $4 a-c)$. Supplementation of cells with ROS scavenger NAC reversed this effectiveness (Fig. 4a-c). Furthermore, overexpression of TAZ diminished intracellular $\mathrm{ROS}$ and $\mathrm{O}_{2}{ }^{-}$levels, reduced the content of MDA, an OS indicator, and restored the expression of Prl8a2 and Prl3c1, as well as ALP activity, while knockdown of TAZ aggravated oxidative impairment of stromal cell differentiation and increased ROS, $\mathrm{O}_{2}{ }^{-}$and MDA levels (Fig. 4d-h, Supplementary Fig. 3a-e).

TAZ prevented oxidative damage to stromal differentiation by restoring cellular antioxidant capacity

Since NOX was the principal source of intracellular $\operatorname{ROS}^{23}$, we explored the regulation of NOX activity by TAZ. Under OS conditions, continuous expression of TAZ did not affect the NOX activity (data not shown), but blockade of TAZ enhanced the NOX activity (Fig. 4i). After transfection with siRNA against TAZ and addition of NOX inhibitor APO, intracellular $\mathrm{O}_{2}{ }^{-}$level was clearly reduced accompanying by the restoration of Prl8a2 and Prl3c1 expression as well as ALP activity (Fig. $4 \mathrm{j}-\mathrm{I}$ ). 
a
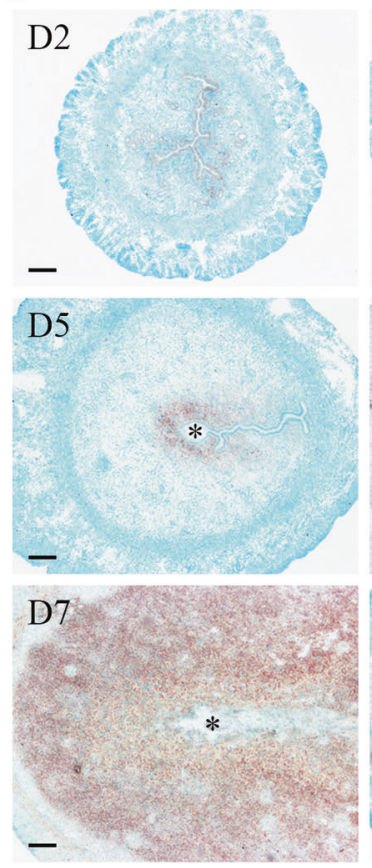

d
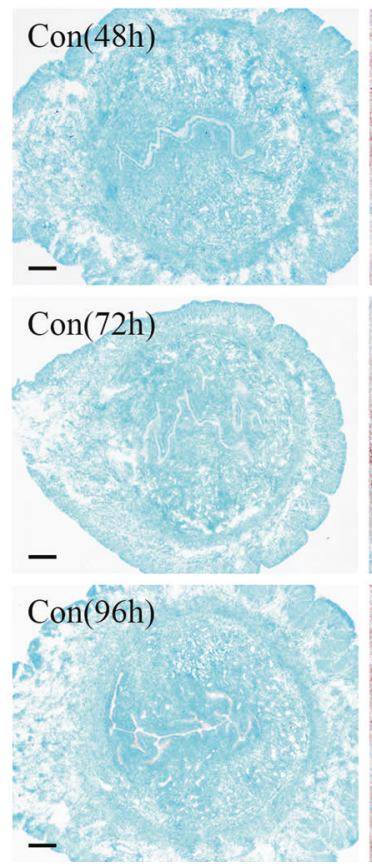
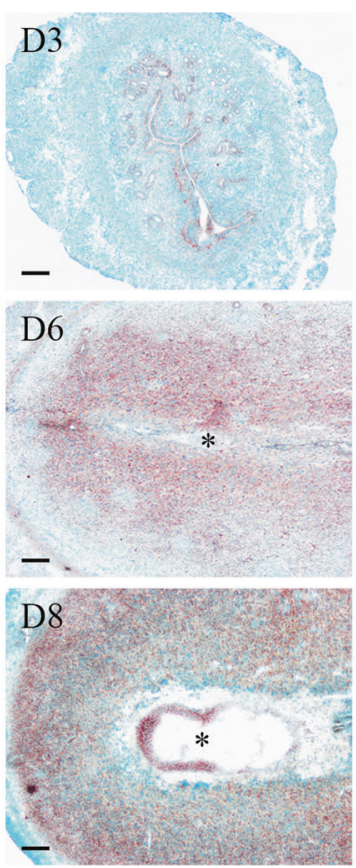

TAZ
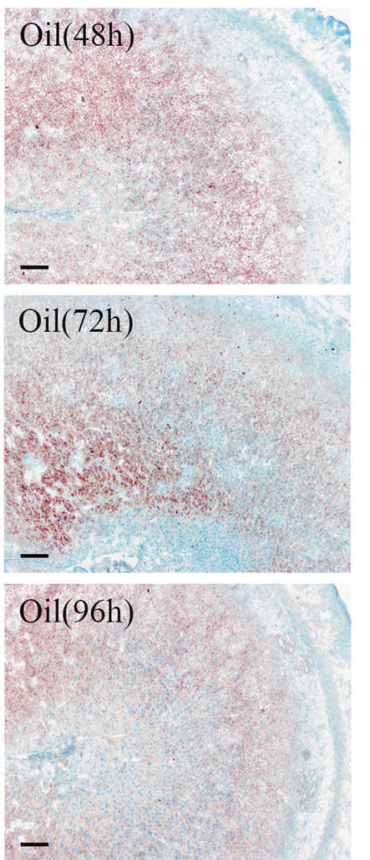

c

e

f b

D1

D2

D3

D4

D5

D6

D7

D8
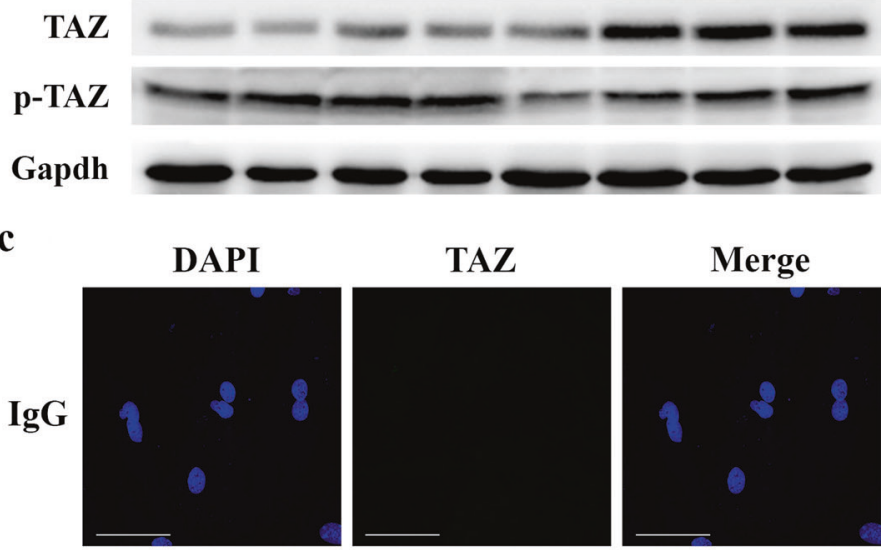

TAZ
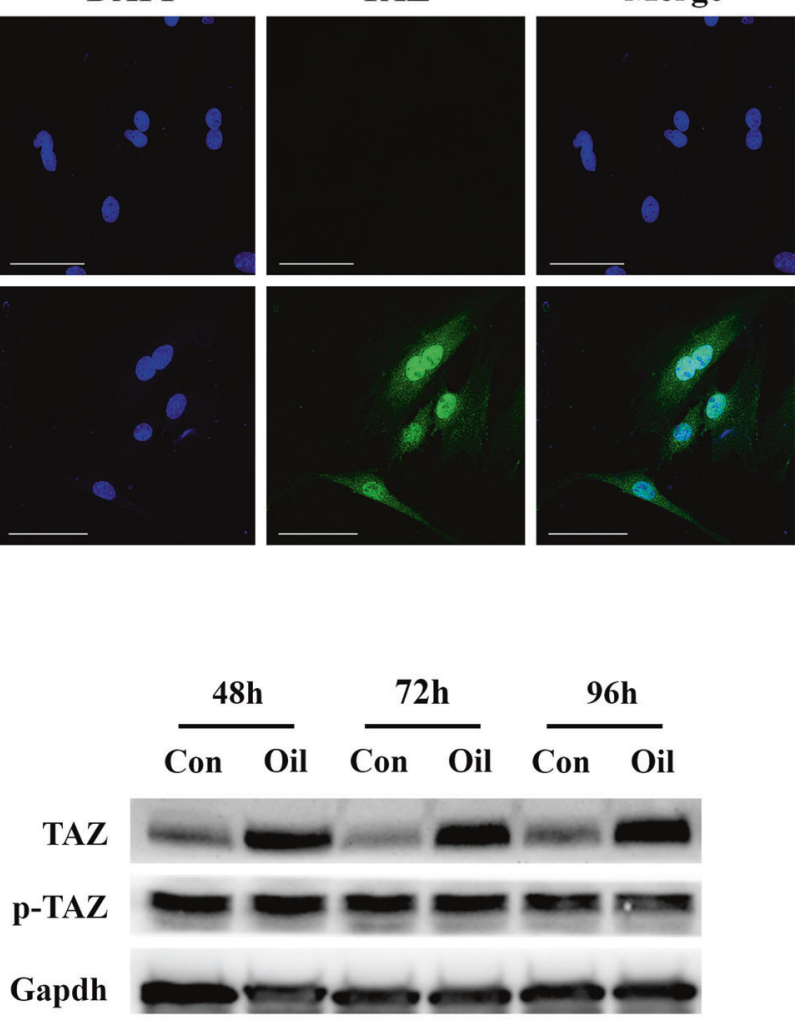

Fig. 1 TAZ expression in uteri and decidual cells. a Localization of uterine TAZ mRNA on days 2, 3, and 5-8 of pregnancy as determined by in situ hybridization. Scale bar, $60 \mu \mathrm{m}$. Asterisks indicate the embryo. b Western blotting analysis of TAZ protein in uteri during early pregnancy. TAZ, total TAZ protein; p-TAZ, phospho-TAZ protein. c Immunofluorescence analysis showed the marked nuclear localization of TAZ protein in decidual cells. d Localization of uterine TAZ mRNA under artificial decidualization. e Western blotting analysis of TAZ protein under artificial decidualization. Con, uninjected uterine horn, which served as the control; Oil, oil-induced decidualization. $\mathbf{f}$ Western blotting analysis of TAZ protein during in vitro decidualization. EP estrogen plus progesterone.

OS is a result of insufficient antioxidant capacity to eliminate excessive $\mathrm{ROS}^{13}$. After exposure to $\mathrm{H}_{2} \mathrm{O}_{2}$, the activities of antioxidant enzymes SOD, CAT and GPX were substantially diminished. However, sustained activation of TAZ improved above antioxidant deficiency, while downregulation of TAZ exacerbated the reduction in antioxidant enzyme activities (Fig. $4 \mathrm{~m}, \mathrm{n}$, Supplementary Fig. 3f, g). The addition of SOD inhibitor DDC,
CAT inhibitor ATZ or GPX inhibitor MS might block the defensive function of overexpressed TAZ on the oxidative damage to stromal cell differentiation (Fig. 4o, p). In addition, TAZ strengthened GR activity followed by the elevation of GSH content and GSH/GSSG ratio, but supplementation with GR inhibitor BCNU and GSH synthesis inhibitor BSO, that reduced GSH content and GSH/ GSSG ratio, hindered the reestablishment of TAZ effects on 
a

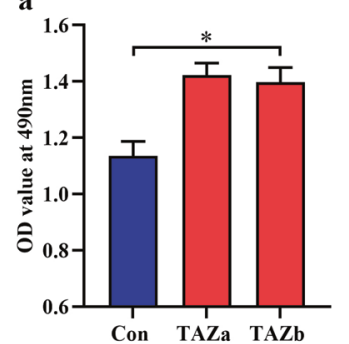

d

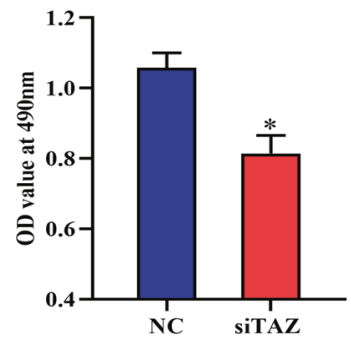

g

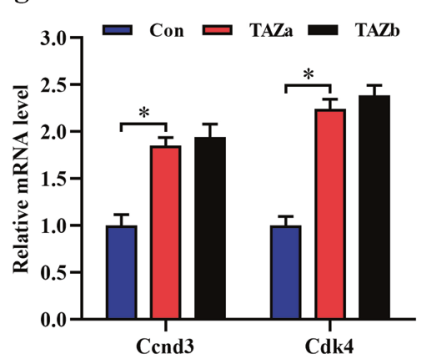

k

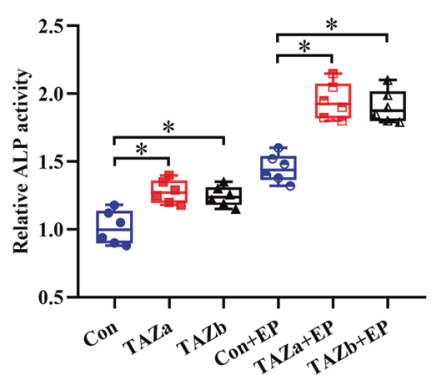

b

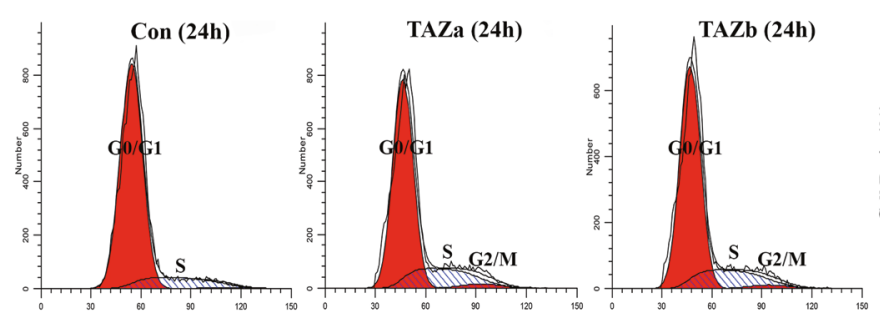

$\mathbf{e}$

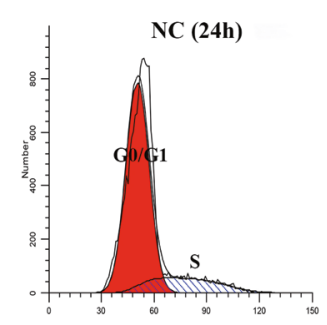

h

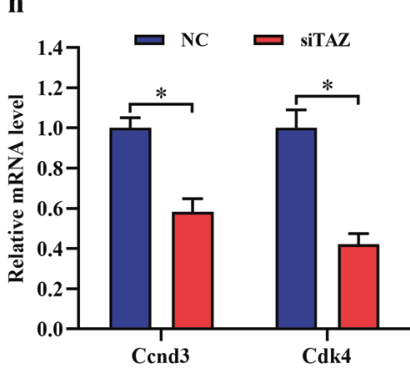

I

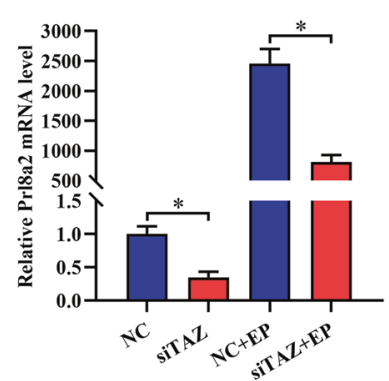

c

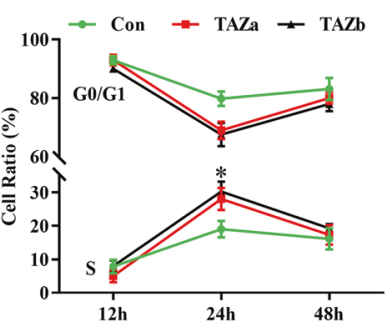

f

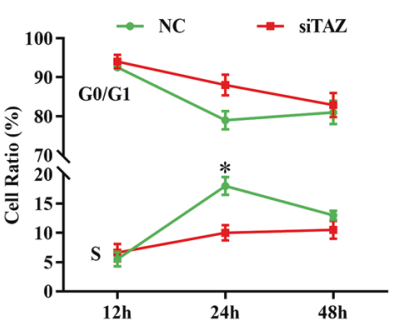

j

i

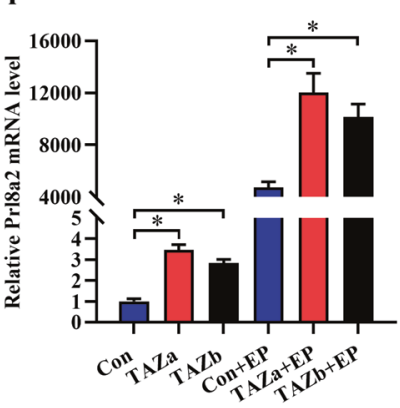

m

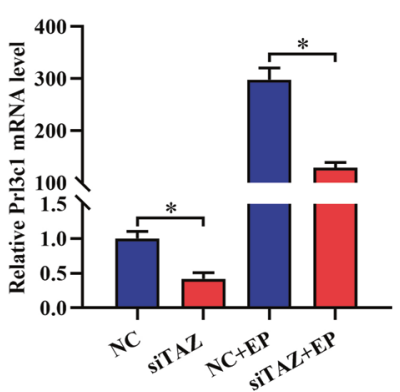

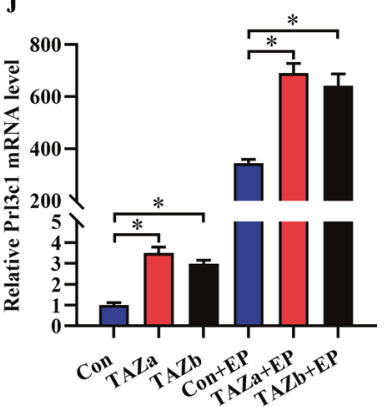

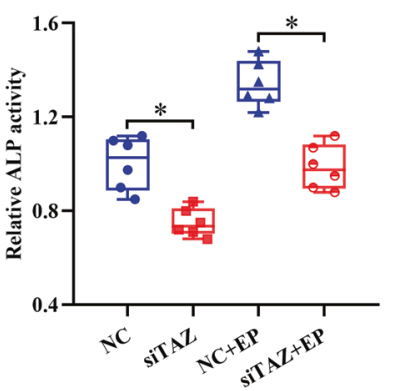

Fig. 2 The role of TAZ in uterine decidualization. a MTS analysis of stromal cell proliferation at $24 \mathrm{~h}$ after introduction of TAZ overexpression plasmid. Con, empty pcDNA3.1 vector; TAZ, TAZ overexpression plasmid. Data are shown the mean \pm SEM. Asterisks denote significance $(P<$ 0.05). b, c Flow cytometry analysis of cell cycle distribution after introduction of TAZ overexpression plasmid for 12,24 , and $48 \mathrm{~h}$. d MTS analysis of stromal cell proliferation after introduction of TAZ siRNA for $24 \mathrm{~h}$. NC, negative control; siTAZ, TAZ siRNA. e, $\mathbf{f}$ Flow cytometry analysis of cell cycle distribution after introduction of TAZ siRNA for 12,24 and $48 \mathbf{h}$. $\mathbf{g}, \mathbf{h}$ Regulation of TAZ overexpression or silencing on Ccnd 3 and Cdk4 expression at $24 \mathrm{~h}$. i-k Constitutive activation of TAZ promoted the expression of Prl8a2 and Prl3c1 and ALP activity at $48 \mathrm{~h}$ with/without estrogen and progesterone. I-n Knockdown of TAZ markedly repressed Prl8a2 and Prl3c1 expression and ALP activity at $48 \mathrm{~h}$ with/without estrogen and progesterone.

disrupted stromal cell differentiation (Fig. 4n-p, Supplementary Fig. 3g-i, Supplementary Fig. 4a-d).

\section{TAZ protected the mitochondrial function of stromal cells under OS}

Mitochondria are crucial for the maintenance of cellular function by governing energy production ${ }^{24}$. Administration of $\mathrm{H}_{2} \mathrm{O}_{2}$ resulted in the impairment of mitochondrial function, as evidenced by the reduction in ATP level and mitochondrial membrane potential together with aberrant mtDNA copy number and elevated mitochondrial $\mathrm{O}_{2}{ }^{-}$level (Fig. 5a-d). Overexpression of TAZ prevented mitochondrial dysfunction, while knockdown of
TAZ exacerbated this disorder (Fig. 5a-d, Supplementary Fig. $5 a-d)$. Further study revealed that TAZ might modulate the activities of mitochondrial respiratory chain complexes I and III whose blockade by ROT and AA abrogated the rescue of Prl8a2 and Prl3c1 expression and ALP activity induced by TAZ overexpression, but no obvious alteration to mitochondrial respiratory chain complex II activity after sustained expression or knockdown of TAZ (Fig. 5e-g, Supplementary Fig. 5e).

TAZ prevented $\mathrm{H}_{2} \mathrm{O}_{2}$ induction of stromal cell apoptosis Previous studies have demonstrated that mitochondrial dysfunction is closely related to cell apoptosis ${ }^{25-27}$. In the context of $\mathrm{H}_{2} \mathrm{O}_{2}$, 
a

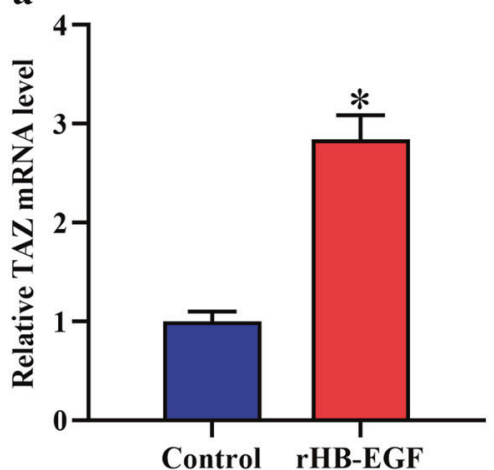

d

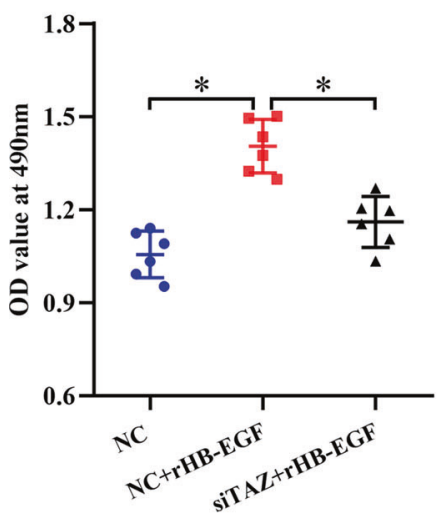

f

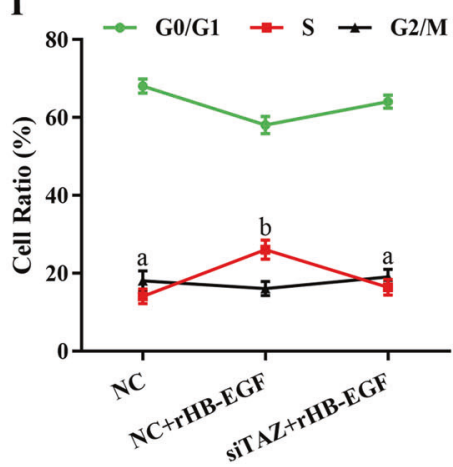

i

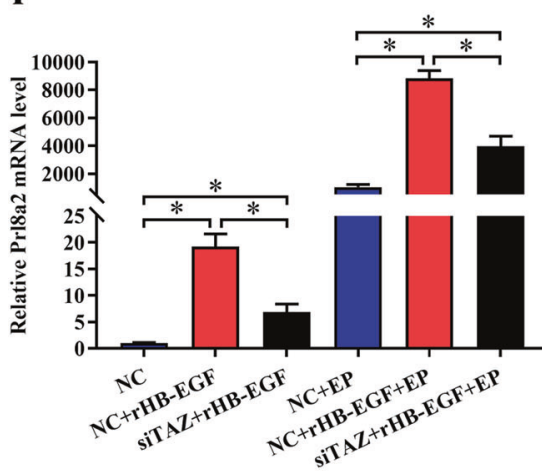

e b

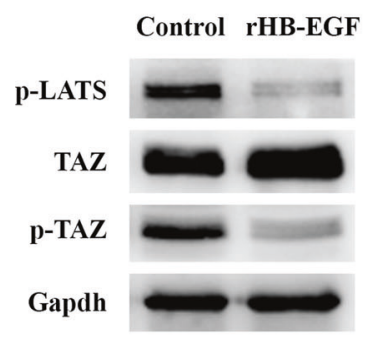

c
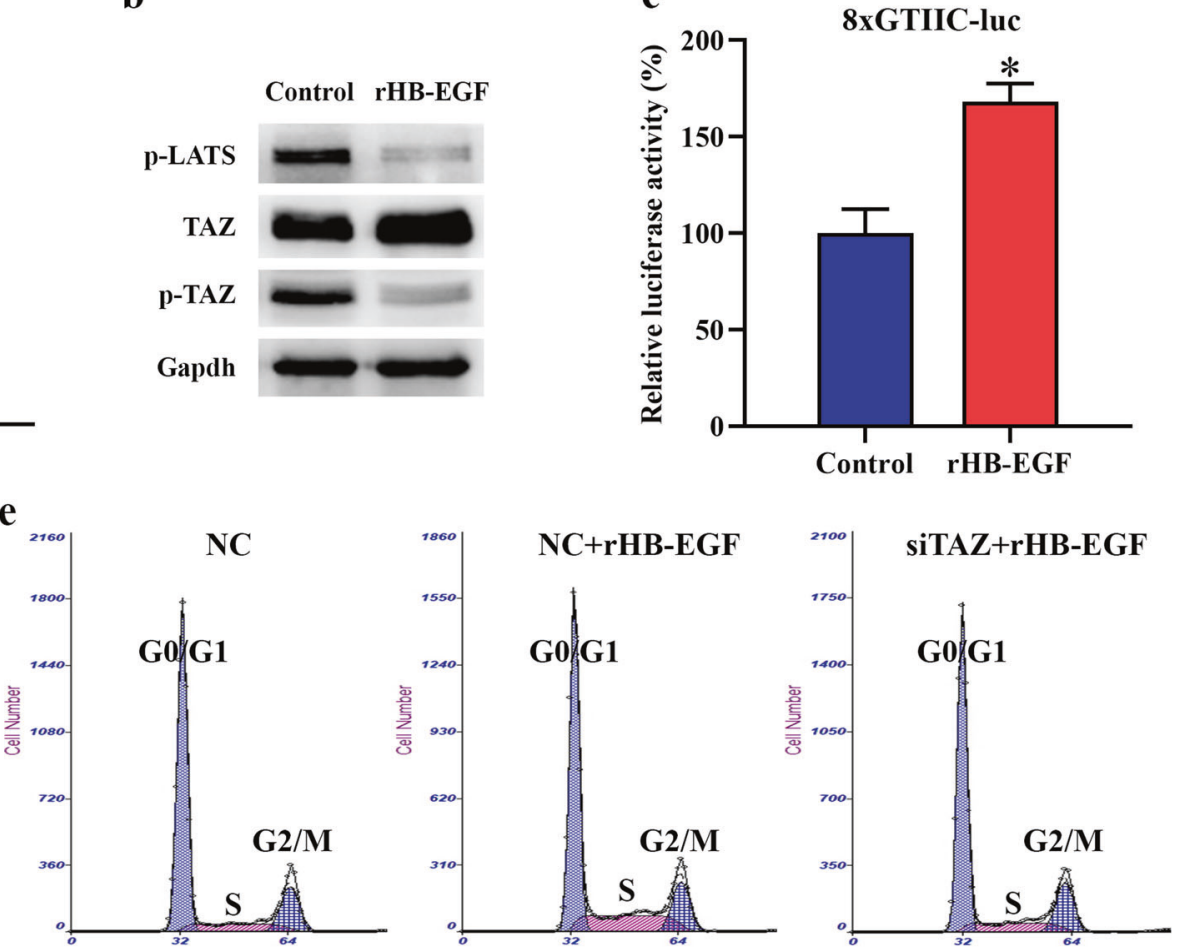

g

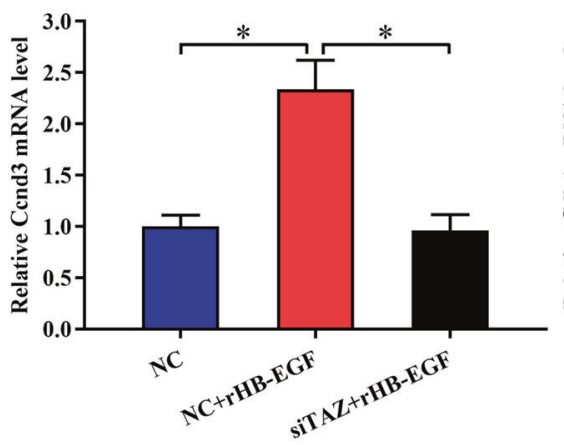

h

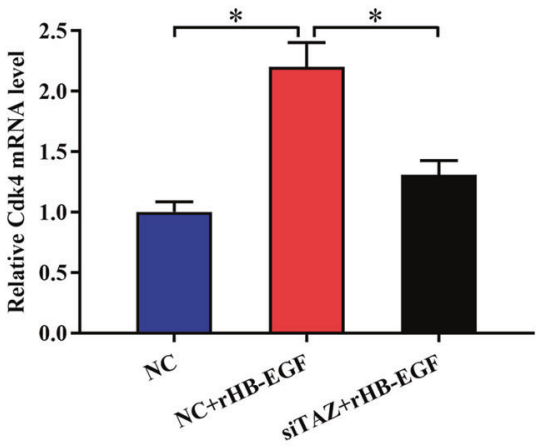

$\mathbf{k}$

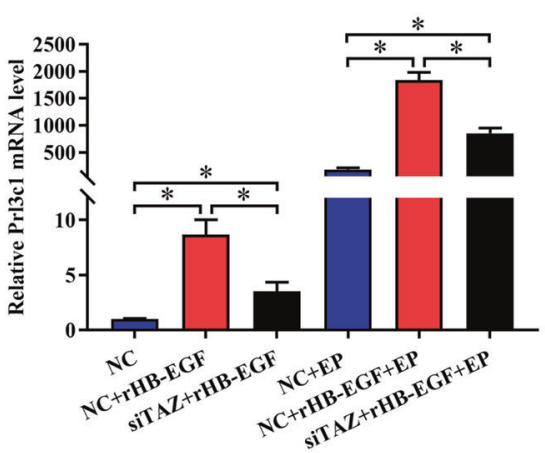

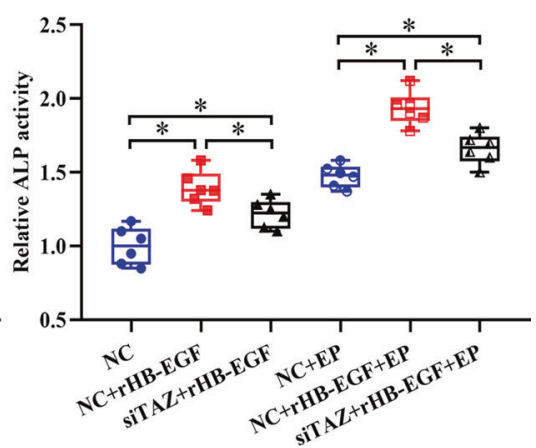

Fig. 3 TAZ mediated HB-EGF function in decidualization. a TAZ expression after supplementation with rHB-EGF for $48 \mathrm{~h}$. $\mathbf{b}$ Regulation of HBEGF on the expression of TAZ and phospho-LATS protein. p-LATS, phospho-LATS protein. c Effect of HB-EGF on TAZ-TEAD transcriptional activity. d Knockdown of TAZ antagonized HB-EGF-induced stromal cell proliferation. e, $\mathbf{f}$ Flow cytometry analysis showed that TAZ mediated HB-EGF function in the regulation of cell cycle. $\mathbf{g}, \mathbf{h}$ Knockdown of TAZ blocked the upregulation of Ccnd3 and Ckd4 that had been induced by HB-EGF. i-k TAZ partially attenuated the induction of HB-EGF on Prl8a2 and Prl3c1 expression and ALP activity. 
a

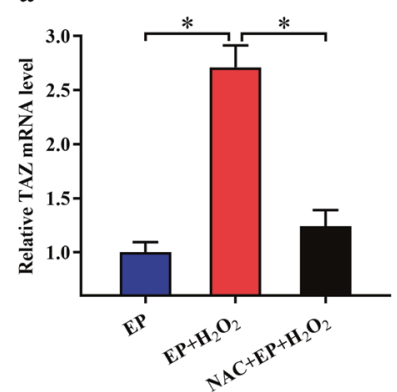

e
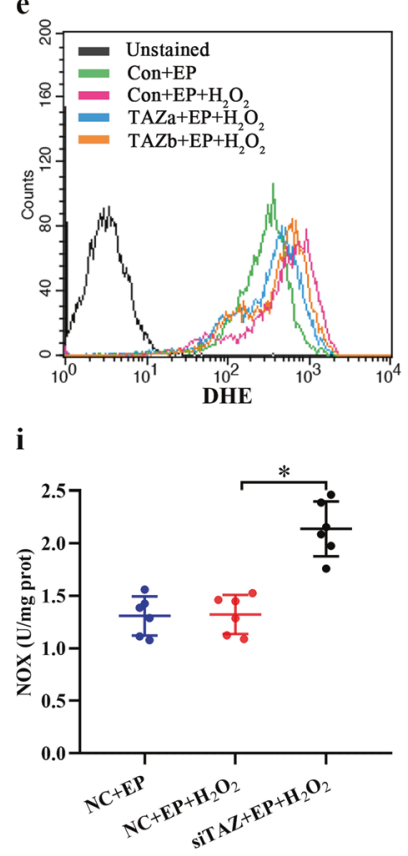

m

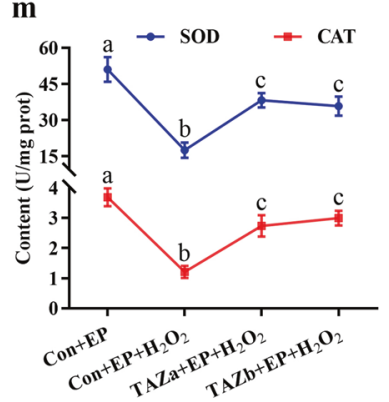

b

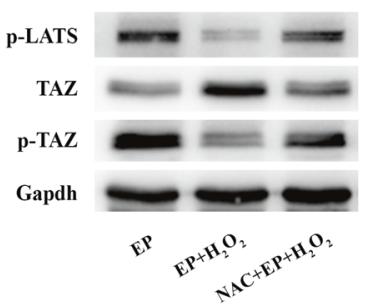

f

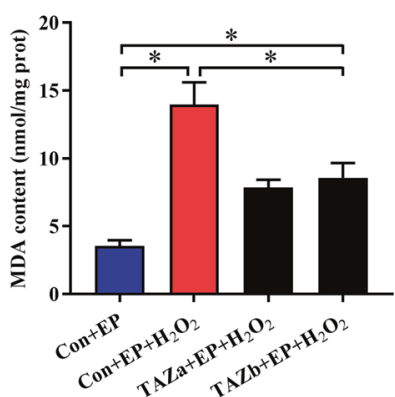

j

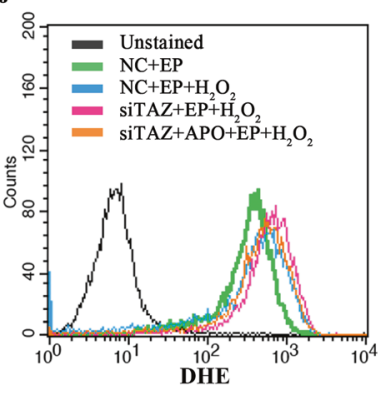

c

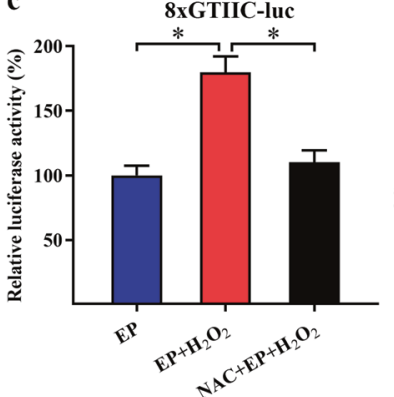

g

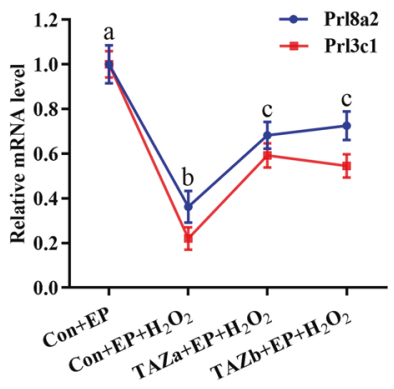

k

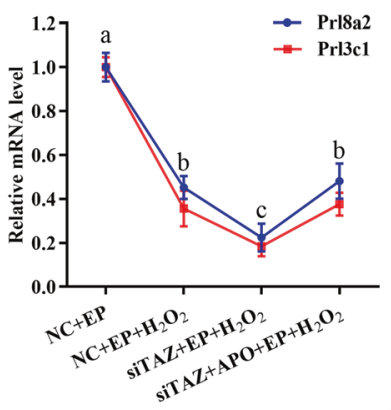

d

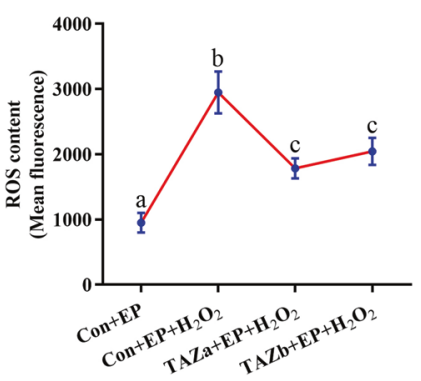

h

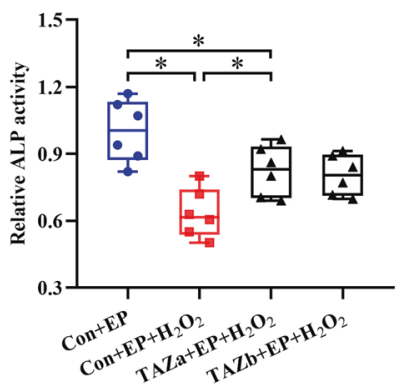

I

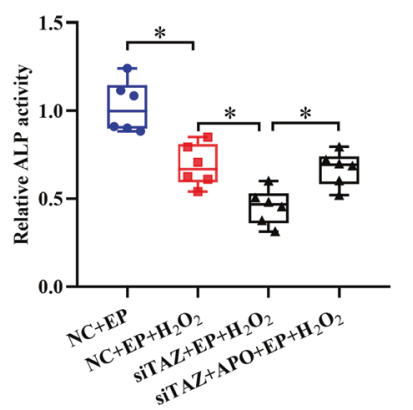

p

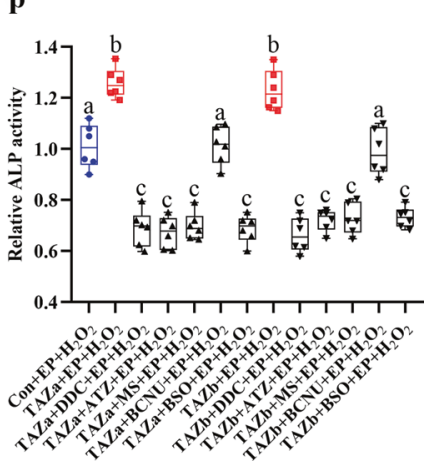

Fig. 4 TAZ protected stromal differentiation from oxidative damage by restoring cellular antioxidant capacity. a TAZ mRNA expression in stromal cells after exposure to $\mathrm{H}_{2} \mathrm{O}_{2}$ with/without NAC. b TAZ and phospho-LATS protein expression after treatment with $\mathrm{H}_{2} \mathrm{O}_{2}$ with/without NAC. c TAZ-TEAD transcriptional activity after treatment with $\mathrm{H}_{2} \mathrm{O}_{2}$ with/without NAC. $\mathbf{d}$-f Overexpression of TAZ attenuated the increase of intracellular $\mathrm{ROS}, \mathrm{O}_{2}{ }^{-}$and MDA levels under OS. $\mathbf{g}, \mathbf{h}$ Overexpression of TAZ protected stromal cell differentiation from oxidative damage. i Knockdown of TAZ increased NOX activity after $\mathrm{H}_{2} \mathrm{O}_{2}$ exposure. $\mathbf{j}$ APO attenuated the increase of intracellular $\mathrm{O}_{2}^{-}$level by TAZ siRNA. $\mathbf{k}$, I APO treatment reversed the repression of TAZ siRNA on stromal cell differentiation under OS. $\mathbf{m}, \mathbf{n}$ Overexpression of TAZ enhanced the activities of SOD, CAT, GPX, and GR under OS. o, p Repression of SOD, CAT, GPX, and GR as well as GSH synthesis by the corresponding inhibitor blocked the protection conferred by TAZ overexpression on stromal cell differentiation under OS. Bars with different letters at the top differ significantly.

continuous expression of TAZ prevented stromal cell apoptosis, whereas depletion of TAZ heightened cell apoptosis rate (Fig. 5h, Supplementary Fig. 5f). Furthermore, overexpression of TAZ weakened Casp3 activity and decreased the mRNA levels of Casp 3 and Bax, and led to the recovery of $\mathrm{Bcl} 2$ expression, while silencing of TAZ exhibited the opposite effects (Fig. 5i, j, Supplementary Fig. 5g, h).

\section{TAZ activated the Nrf2/ARE/Foxo1 pathway under OS}

It is well known that Nrf2 and Foxo1 are important modulators of cellular antioxidant defense ${ }^{28,29}$. Under OS, Nrf2 and Foxo1 expression levels, as well as Nrf2 nuclear translocation, were obviously diminished, but sustained activation of TAZ reversed these effects (Fig. 6a-e). Moreover, TAZ enhanced the transcriptional activity of Nrf2 (Fig. 6f, g). Blockade of Nrf2 

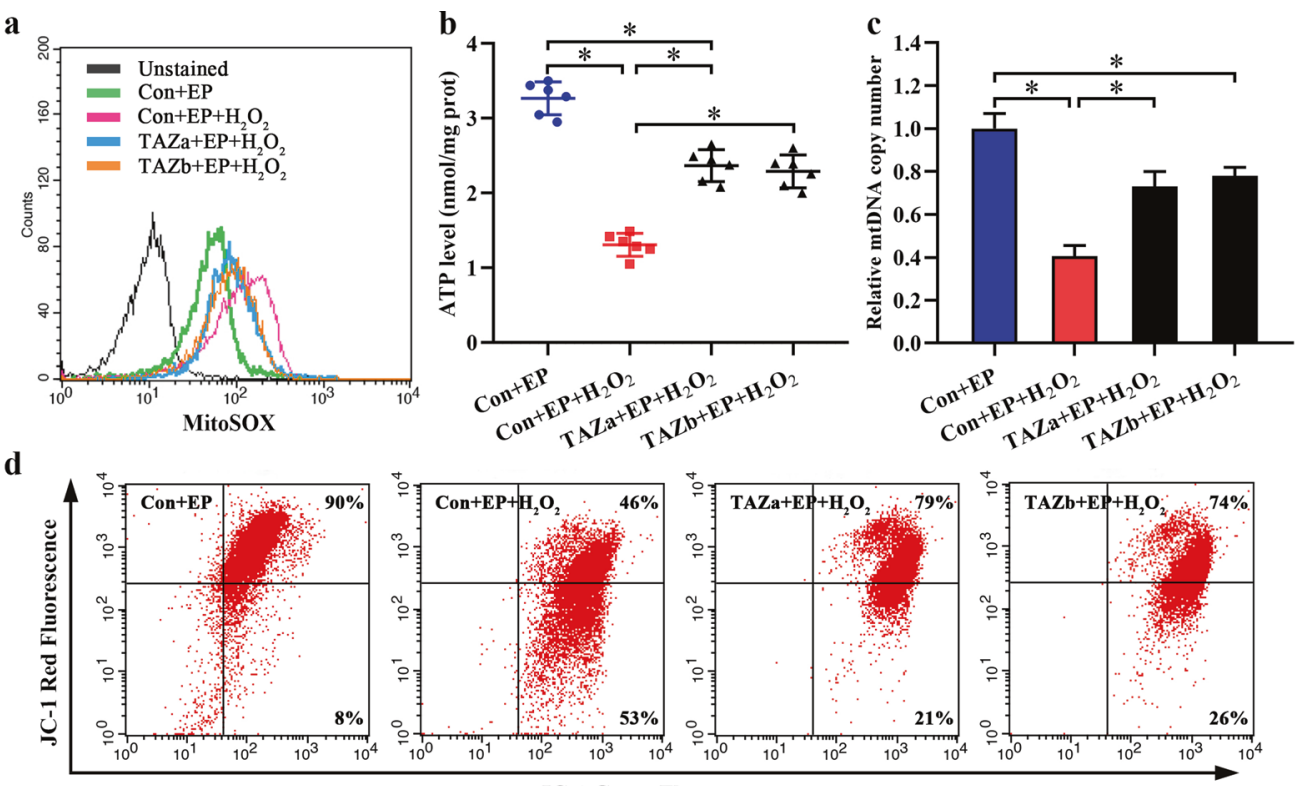

e f
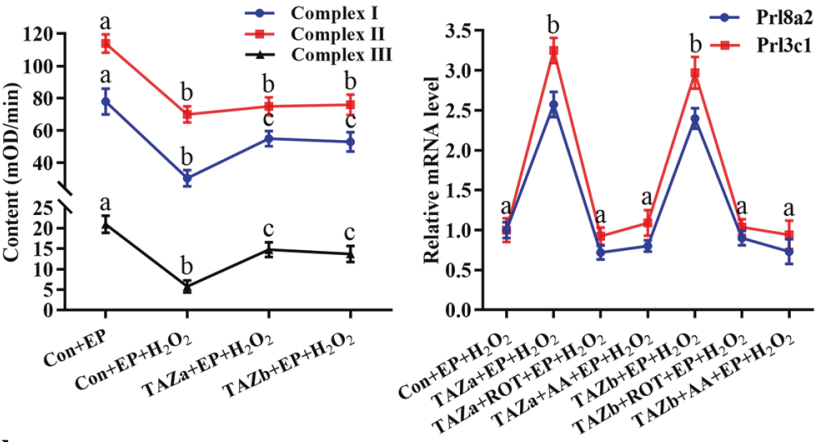

g

h
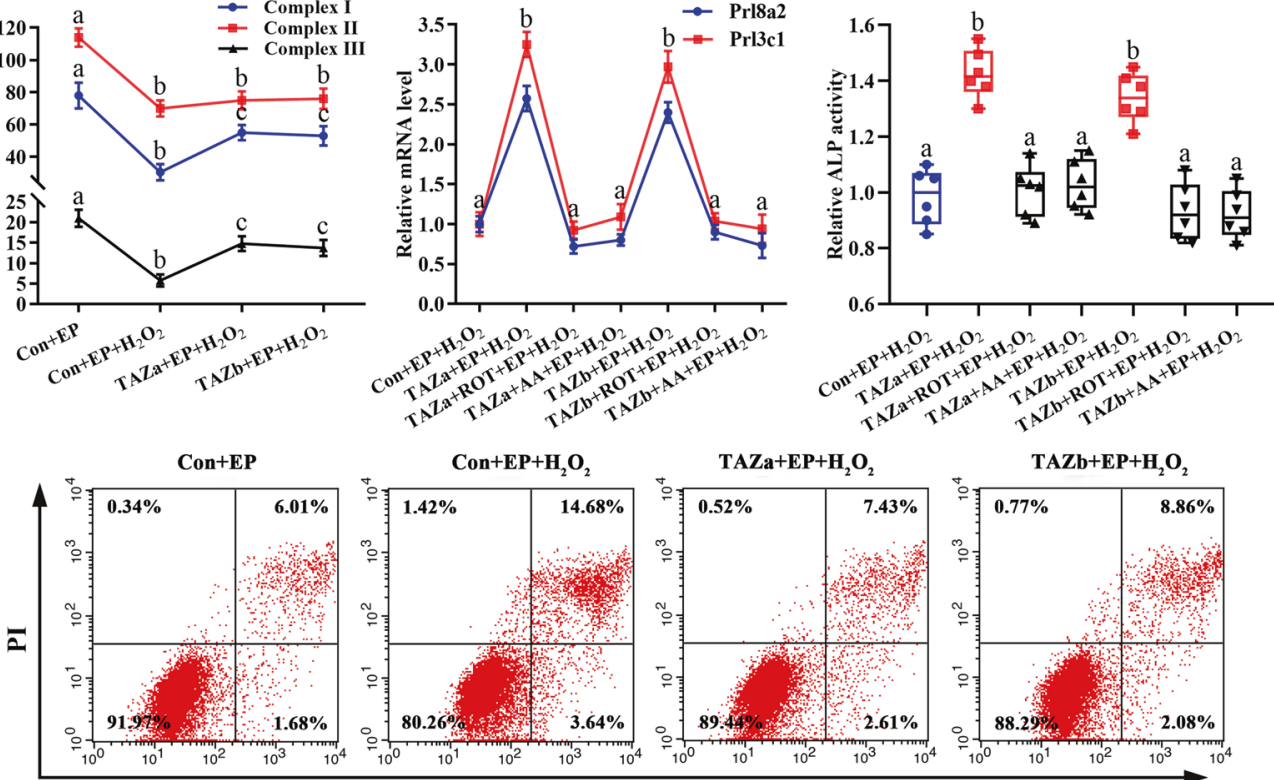

i

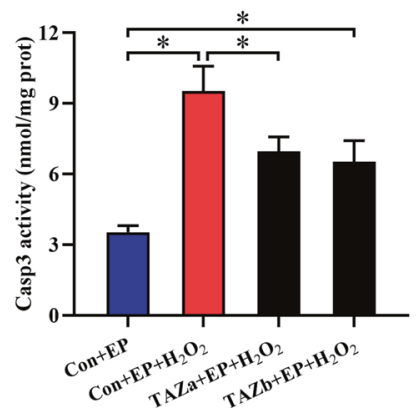

Annexin V-FITC

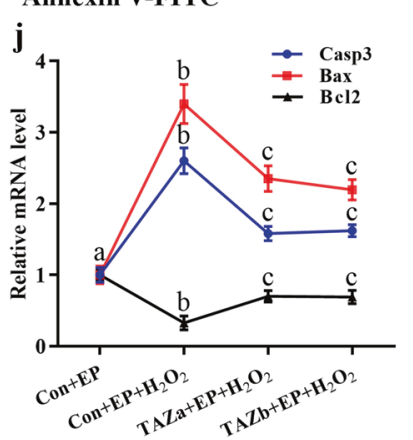

Fig. 5 TAZ prevented mitochondrial dysfunction and stromal cell apoptosis under OS. a Overexpression of TAZ decreased mitochondrial $\mathrm{O}_{2}^{-}$level under OS. b-d Overexpression of TAZ hindered $\mathrm{H}_{2} \mathrm{O}_{2}$-induced damage to ATP level, mtDNA copy number, and mitochondrial membrane potential. e Effects of TAZ overexpression on the activities of mitochondrial respiratory chain complexes I, II, and III under OS. $\mathbf{f}, \mathbf{g}$ ROT and AA abrogated the protection conferred by TAZ overexpression on stromal cell differentiation under OS. $\mathbf{h}$ Overexpression of TAZ blocked stromal cell apoptosis under OS. $\mathbf{i}$, j Overexpression of TAZ weakened Casp3 activity, reduced the levels of Casp3 and Bax mRNA and induced the recovery of $\mathrm{BCl} 2$ expression under OS. 


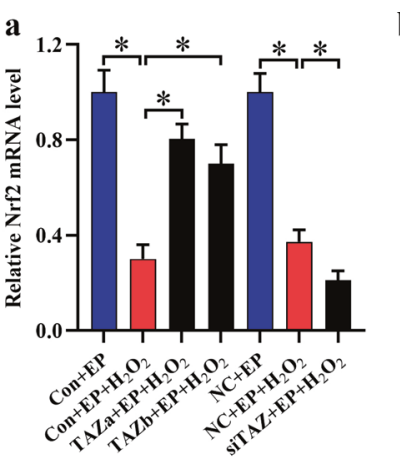

b
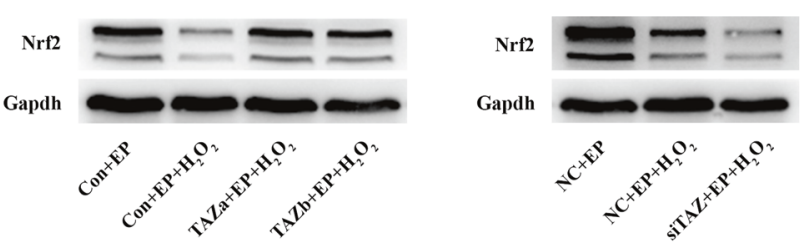

e
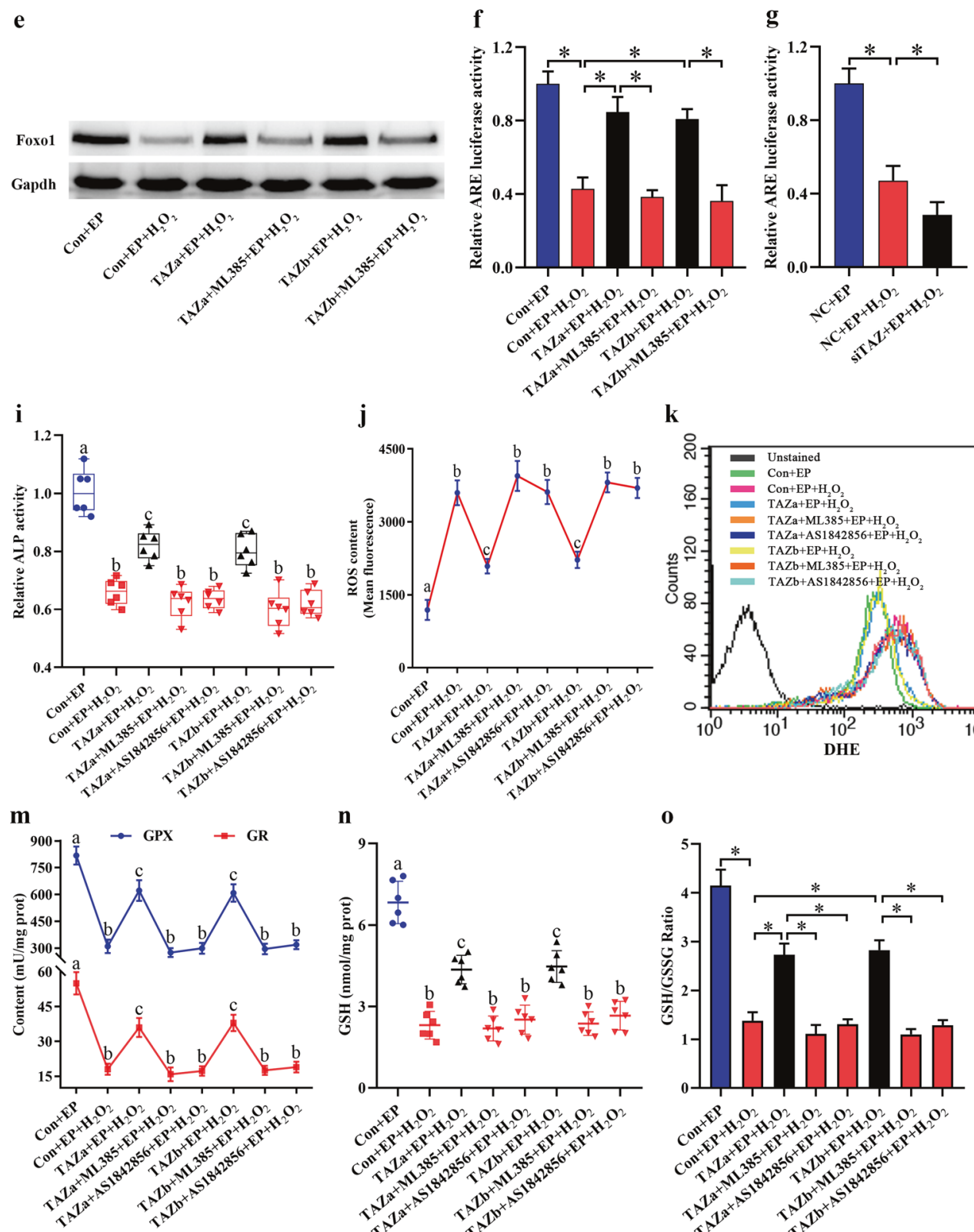

j

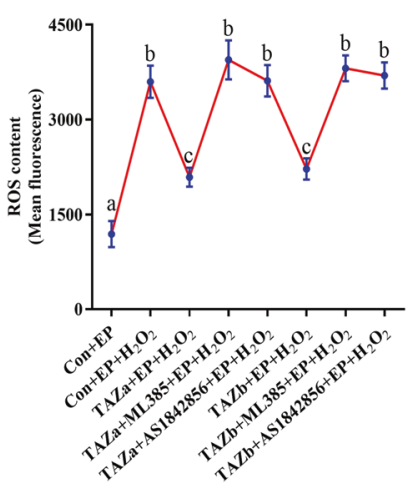

n

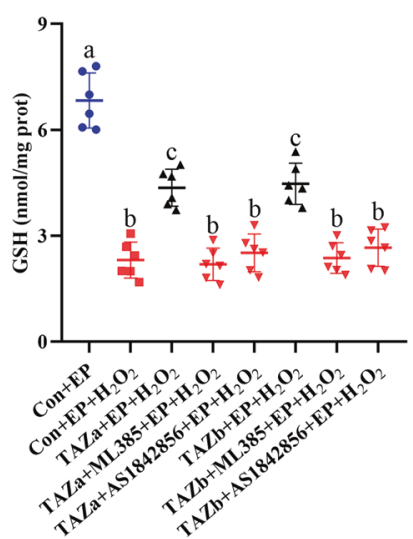

k
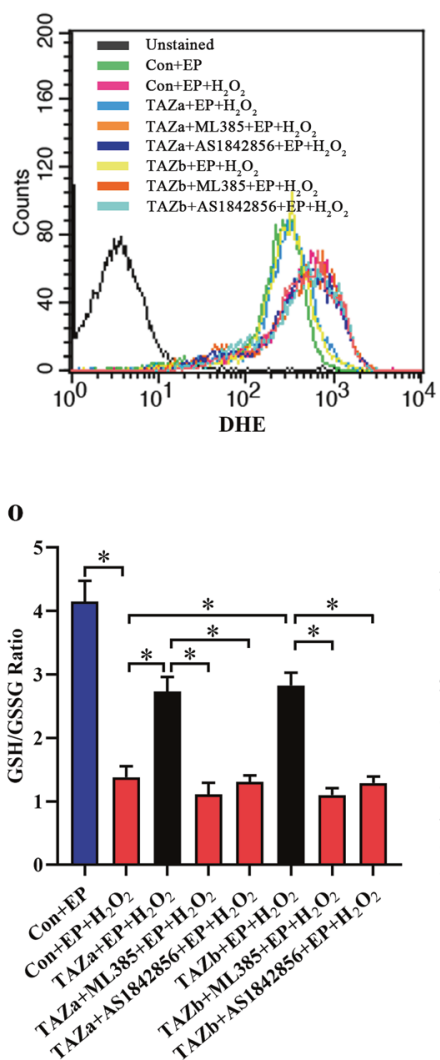

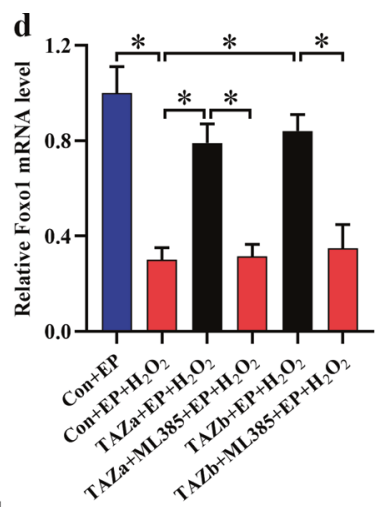

h

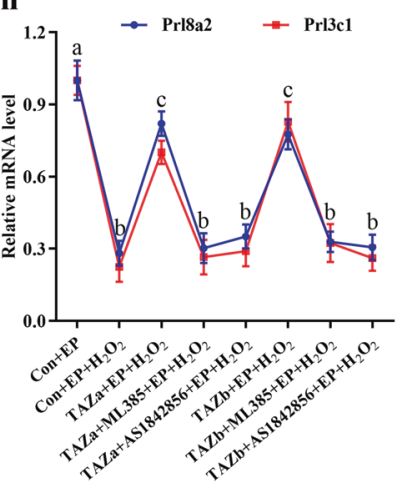

1

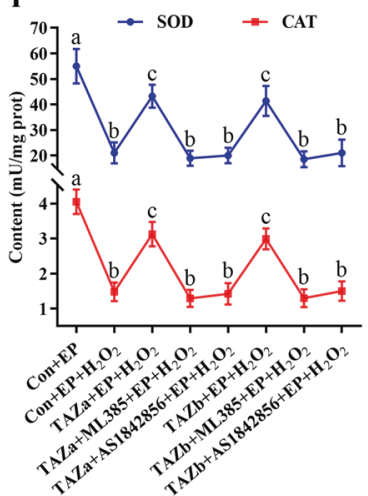

p

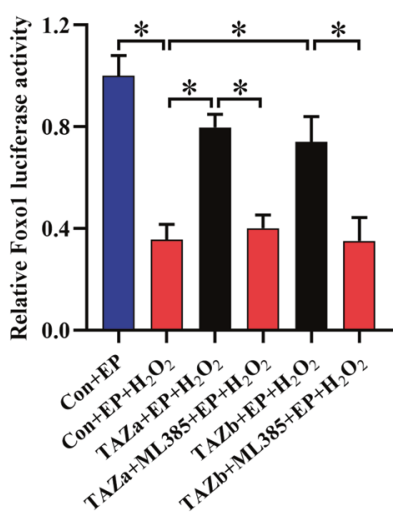

Fig. 6 TAZ restored cellular antioxidant capacity via the Nrf2-ARE-Foxo1 pathway under OS. a-c Effects of TAZ overexpression or knockdown on the mRNA and protein levels of Nrf2. d, e Effects of TAZ overexpression on the mRNA and protein levels of Foxo1 with/without Nrf2 inhibitor ML385. f, g Effects of TAZ overexpression or knockdown on Nrf2 transcriptional activity. $\mathbf{h}$, i Blockade of Nrf2 and Foxo1 with the corresponding inhibitor abrogated the protection conferred by TAZ overexpression on stromal cell differentiation under OS. $\mathbf{j}$, $\mathbf{k}$ Repression of Nrf2 and Foxo1 impeded the reduction in intracellular ROS and $\mathrm{O}_{2}{ }^{-}$levels caused by TAZ overexpression. I-o Blockade of Nrf2 and Foxo1 abolished the ability of TAZ to rescue the activities of SOD, CAT, GPX and GR as well as GSH content and GSH/GSSG ratio. $\mathbf{p}$ Effects of TAZ overexpression on Foxo1 promoter activity in the absence or presence of ML385. 
and Foxo1 with the corresponding inhibitor abrogated TAZ protection of stromal cell differentiation to oxidative damage, antagonized TAZ attenuation of intracellular ROS and impeded the rescue of TAZ on the enzymatic activities of SOD, CAT, GPX and GR as well as GSH content and GSH/GSSG ratio (Fig. 6h-o). Further evidence revealed that addition of Nrf2 inhibitor ML385 abrogated the ability of TAZ to rescue the mRNA and protein levels of Foxo1 under OS (Fig. 6d, e). Analysis of Foxo1 promoter region exhibited the presence of antioxidant response element (ARE) harboring an Nrf2-binding site. Overexpression of TAZ restored the luciferase activity of Foxo1 reporter plasmid under $\mathrm{OS}$, but this restoration was blocked by Nrf2 inhibitor ML385 (Fig. 6p).

\section{DISCUSSION}

TAZ is required for spermatogenesis and fertilization, but its physiological role in uterine decidualization has not been studied. In this study, we found that TAZ was clearly observed in decidual cells. In addition, abundant nuclear TAZ protein was identified in human endometrial stromal cells that underwent expansive proliferation and then differentiated into decidual cells, a process known as decidualization ${ }^{2,12}$. Stromal cell proliferation is the first step of decidualization ${ }^{30}$. Overexpression of TAZ drived stromal cell proliferation, while silencing of TAZ had the opposite effect. Consistently, TAZ might exert proliferative activity in mammary and periodontal ligament stem cells, and stimulate liver regeneration by increasing hepatocyte proliferation ${ }^{31-33}$. It is known that cellular proliferation is accompanied by the alteration of cell cycle $^{34}$. TAZ promoted the G1/S phase transition which was adjusted by $\mathrm{Ccnd} 3$, a G1 phase modulator of stromal cell proliferation ${ }^{2,35}$. Deficiency of $\mathrm{Ccnd} 3$ resulted in defective decidualization concomitant with the impairment of stromal cell differentiation ${ }^{2,20,35}$. Constitutive activation of TAZ increased the expression or activity of Prl8a2, Prl3c1 and ALP, reliable markers for stromal differentiation, whereas knockdown of TAZ led to the opposite effects. Together these observations suggest that TAZ plays an important role in uterine decidualization through $\mathrm{Cond} 3$.

HB-EGF is a fundamental modulator of decidualization ${ }^{20,21}$. Ablation of HB-EGF led to compromised pregnancy outcomes with defective decidual development ${ }^{20,21,36}$. Adjunction of exogenous rHB-EGF facilitated the expression of TAZ total protein and diminished the phosphorylation of TAZ as well as LATS which was one of core components for Hippo pathway and its activation resulted in cytoplasmic sequestration of phosphorylated TAZ, while deletion of LATS heightened nuclear enrichment of $T_{A Z}{ }^{22,37}$. Concurrently, after import into the nucleus, unphosphorylated TAZ executes its transcriptional activity by interacting with TEAD transcription factors ${ }^{22}$. Under in vitro decidualization conditions, HB-EGF remarkably enhanced the transcriptional activity of TAZ-TEAD. Together these data indicate that HB-EGF activates TAZ-TEAD by repressing LATS phosphorylation. Further analysis revealed that inhibition of TAZ abrogated HB-EGF induction of stromal cell proliferation and differentiation, indicating that TAZ serves as a downstream target of HB-EGF in decidualization. Previously reported data evidenced that HB-EGF might boost the expression of Ccnd3 which was necessary for HB-EGF-driven decidualization $^{20}$. Knockdown of TAZ abolished the promotion of HBEGF on Ccnd3, suggesting that TAZ functions as an important intermediary between HB-EGF and Cond3.

Intracellular ROS that was not scavenged by the intrinsic antioxidant system induced OS, which impaired decidualization $^{13-15}$. As one of the most important cellular antioxidants ${ }^{14}$, GSH was shown to have diminished content under OS, while activation of TAZ improved the level of GSH whose synthesis blockade by BSO impeded the defense of TAZ against oxidative damage to stromal cell differentiation. Previous studies have shown that GPX might oxidize GSH to GSSG in metabolizing $\mathrm{H}_{2} \mathrm{O}_{2}$ into water, while GR diminished GSSG back to $\mathrm{GSH}^{14}$. In the present study, TAZ enhanced the enzymatic activities of GPX and $G R$, whose inhibition disrupted the protective effect of TAZ on stromal cell differentiation under OS. Together these observations suggest that TAZ may defend against oxidative damage to uterine decidualization via GSH. Furthermore, TAZ might modulate the activities of SOD and CAT which were two important antioxidant enzymes in uterine decidualization and catalyzed the dismutation of $\mathrm{O}_{2}{ }^{-}$to $\mathrm{H}_{2} \mathrm{O}_{2}$ or the conversion of $\mathrm{H}_{2} \mathrm{O}_{2}$ to water $^{14,38}$. Collectively, these results reveal that TAZ prevents oxidative damage to stromal cell differentiation by restoring antioxidant capacity.

After translocation into the nucleus, TAZ may modulate gene transcription ${ }^{39}$. Under OS, TAZ promoted the expression of Nrf2 which was a redox-sensitive transcription factor and its loss caused an increase in intracellular ROS due to defective antioxidant capability ${ }^{40}$. Repression of $\mathrm{Nrf2}$ might annihilate the defense of TAZ on oxidative damage to stromal cell differentiation, indicating that Nrf2 is a downstream target of TAZ in antioxidant function. Further analysis revealed that TAZ induced nuclear translocation of Nrf2, where it might bind to the ARE of target gene promoter region ${ }^{28}$. Treatment with Nrf2 inhibitor ML385 abrogated the increased luciferase intensity of Foxo1 reporter plasmid induced by TAZ overexpression and impeded the TAZ stimulation of Foxo1, which was an important modulator of cellular antioxidant defense and its ablation in uteri caused infertility owing to the alteration of epithelial integrity along with aberrant decidual response $\mathrm{e}^{29,41}$. Moreover, blockade of Foxo1 abolished the ability of TAZ to defend stromal cell differentiation from oxidative disruption which was followed by the enhancement of intracellular ROS levels. Together these observations suggest that TAZ activates the Nrf2/ARE/Foxo1/ROS pathway to protect stromal cell differentiation against oxidative damage.

NOX is the principal source of intracellular $\operatorname{ROS}^{23}$. Attenuation of TAZ led to an obvious elevation in NOX activity along with abundant accumulation of intracellular ROS. Impediment of NOX by APO attenuated the damage to stromal cell differentiation elicited by TAZ knockdown concomitant with the reduced $\mathrm{O}_{2}{ }^{-}$ level, indicating the importance of TAZ in mediating ROS sources via NOX. In addition, as crucial organelles for energy production, mitochondria generate ROS, but excessive ROS can attack mitochondria, resulting in mitochondrial dysfunction as evidenced by the reduction in ATP synthesis, mitochondrial membrane potential and respiratory enzyme activities along with the elevation of mitochondrial ROS levels ${ }^{25,26,42}$. Under OS conditions, TAZ prevented mitochondrial dysfunction in stromal cells. Furthermore, mtDNA is vulnerable to excessive ROS owing to the lack of protective histones ${ }^{25,26}$. After exposure to $\mathrm{H}_{2} \mathrm{O}_{2}$, TAZ ameliorated oxidative damage to mtDNA copy number, reinforcing the role of TAZ in protecting mitochondrial function. Further analysis showed that mitochondrial dysfunction caused cell apoptosis which was mediated by the apoptosis executor Casp3, pro-apoptotic effector Bax and anti-apoptotic $B \mathrm{cl} 2^{25-27}$. During in vitro decidualization, TAZ prevented the apoptosis of stromal cells induced by $\mathrm{OS}$ through raising $\mathrm{BCl} 2$ expression and suppressing the upregulation of Casp3 and Bax.

In conclusion, TAZ might play an important role in uterine decidualization via $\mathrm{Ccnd} 3$ and $\mathrm{Cdk} 4$ in response to HB-EGF. Furthermore, TAZ ameliorated the oxidative damage to stromal cell differentiation by enhancing antioxidant capacity dependent on the Nrf2/ARE//Foxo1 pathway, recovering mitochondrial function and repressing cell apoptosis (Fig. 7). 


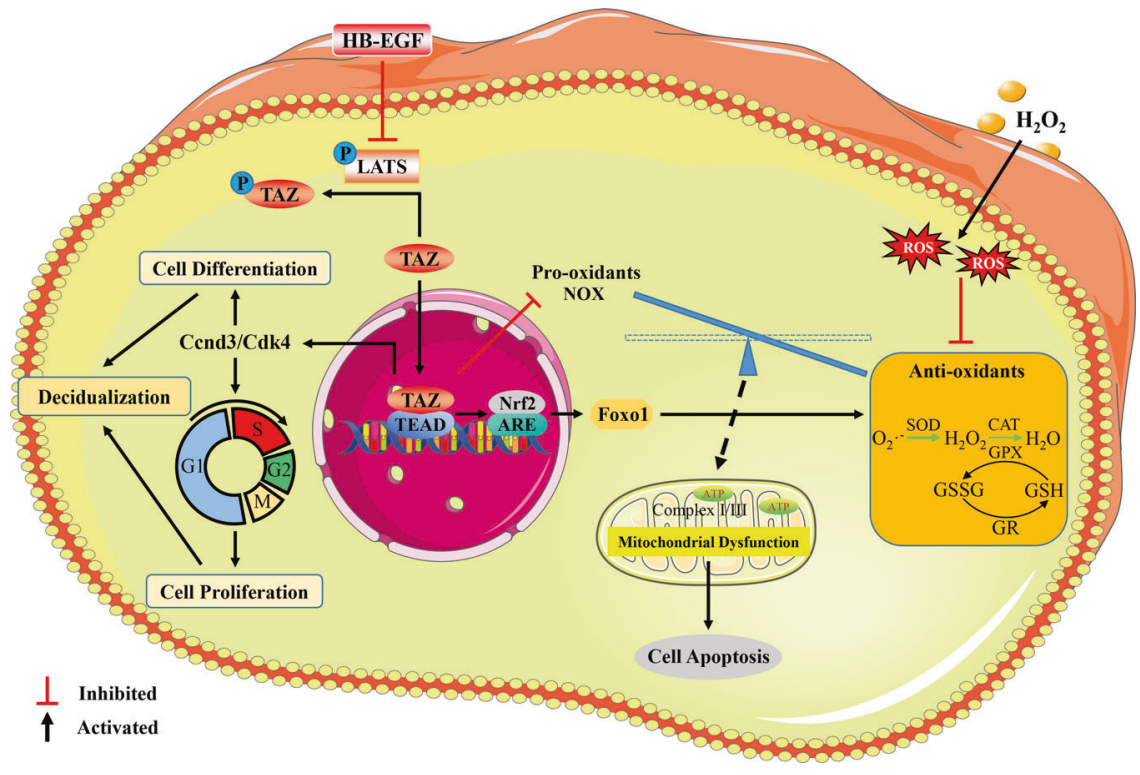

Fig. 7 Schematic depiction of TAZ function and regulation in decidualization. TAZ might exert an important role in uterine decidualization via Ccnd3 and Cdk4 responsiveness to HB-EGF. Furthermore, TAZ ameliorated oxidative damage to stromal differentiation by enhancing cellular antioxidant capacity dependent on Nrf2/ARE/Foxo1 pathway, restoring mitochondrial function and inhibiting cell apoptosis.

\section{DATA AVAILABILITY}

The data that support the findings of this study are available on request to the corresponding author.

\section{REFERENCES}

1. Dey, S. K. et al. Molecular cues to implantation. Endocr. Rev. 25, 341-373 (2004).

2. Zhang, S. et al. Physiological and molecular determinants of embryo implantation. Mol. Asp. Med. 34, 939-980 (2013).

3. Strug, M. R. et al. RBPJ mediates uterine repair in the mouse and is reduced in women with recurrent pregnancy loss. FASEB J. 32, 2452-2466 (2018).

4. Hong, J. H. et al. TAZ, a transcriptional modulator of mesenchymal stem cell differentiation. Science 309, 1074-1078 (2005).

5. Yu, F. X., Zhao, B. \& Guan, K. L. Hippo pathway in organ size control, tissue homeostasis, and cancer. Cell 163, 811-828 (2015).

6. $\mathrm{Yu}, \mathrm{B}$. et al. PGC-1a controls skeletal stem cell fate and bone-fat balance in osteoporosis and skeletal aging by inducing TAZ. Cell Stem Cell 23, 615-623 (2018).

7. Hossain, Z. et al. Glomerulocystic kidney disease in mice with a targeted inactivation of Wwtr1. Proc. Natl Acad. Sci. USA 104, 1631-1636 (2007).

8. Nishioka, N. et al. The Hippo signaling pathway components Lats and Yap pattern Tead4 activity to distinguish mouse trophectoderm from inner cell mass. Dev. Cell 16, 398-410 (2009)

9. Jeong, M. G. et al. Transcriptional coactivator with PDZ-binding motif is required to sustain testicular function on aging. Aging cell 16, 1035-1042 (2017).

10. Dingare, C. et al. The Hippo pathway effector Taz is required for cell morphogenesis and fertilization in zebrafish. Development 145, dev167023 (2018).

11. Yi, X. et al. The effector of Hippo signaling, Taz, is required for formation of the micropyle and fertilization in zebrafish. PLoS Genet. 15, e1007408 (2019).

12. Strakova, Z., Reed, J. \& Ihnatovych, I. Human transcriptional coactivator with PDZbinding motif (TAZ) is downregulated during decidualization. Biol. Reprod. 82, 1112-1118 (2010).

13. Ruder, E. H., Hartman, T. J., Blumberg, J. \& Goldman, M. B. Oxidative stress and antioxidants: exposure and impact on female fertility. Hum. Reprod. Update 14, 345-357 (2008).

14. Agarwal, A., Aponte-Mellado, A., Premkumar, B. J., Shaman, A. \& Gupta, S. The effects of oxidative stress on female reproduction: a review. Reprod. Biol. Endocrinol. 10, 49 (2012).

15. Yu, H. F. et al. HB-EGF ameliorates oxidative stress-mediated uterine decidualization damage. Oxid. Med. Cell. Longev. 2019, 6170936 (2019).

16. Gandhirajan, R. K. et al. Cysteine S-glutathionylation promotes stability and activation of the Hippo downstream effector transcriptional co-activator with PDZ-binding motif (TAZ). J. Biol. Chem. 291, 11596-11607 (2016).

17. $\mathrm{Yu}, \mathrm{H}$. F. et al. Malic enzyme 1 is important for uterine decidualization in response to progesterone/CAMP/PKA/HB-EGF pathway. FASEB J. 34, 3820-3837 (2020).

18. Ou, X. H. et al. Maternal insulin resistance causes oxidative stress and mitochondrial dysfunction in mouse oocytes. Hum. Reprod. 27, 2130-2145 (2012).
19. Zhang, X. et al. nm23 regulates decidualization through the PI3K-Akt-mTOR signaling pathways in mice and humans. Hum. Reprod. 31, 2339-2351 (2016).

20. Tan, Y. et al. HB-EGF directs stromal cell polyploidy and decidualization via cyclin D3 during implantation. Dev. Biol. 265, 181-195 (2004).

21. Chobotova, K. et al. Heparin-binding epidermal growth factor and its receptors mediate decidualization and potentiate survival of human endometrial stromal cells. J. Clin. Endocrinol. Metab. 90, 913-919 (2005).

22. Reggiani, F., Gobbi, G., Ciarrocchi, A. \& Sancisi, V. YAP and TAZ are not identical twins. Trends Biochem. Sci. 46, 154-168 (2021).

23. Dan Dunn, J., Alvarez, L. A., Zhang, X. \& Soldati, T. Reactive oxygen species and mitochondria: a nexus of cellular homeostasis. Redox Biol. 6, 472485 (2015).

24. Roger, A. J., Muñoz-Gómez, S. A. \& Kamikawa, R. The origin and diversification of mitochondria. Curr. Biol. 27, R1177-R1192 (2017).

25. Agarwal, A., Gupta, S., Sekhon, L. \& Shah, R. Redox considerations in female reproductive function and assisted reproduction: from molecular mechanisms to health implications. Antioxid. Redox Signal. 10, 1375-1403 (2008).

26. Bhargava, P. \& Schnellmann, R. G. Mitochondrial energetics in the kidney. Nat. Rev. Nephrol. 13, 629-646 (2017).

27. Singh, R., Letai, A. \& Sarosiek, K. Regulation of apoptosis in health and disease: the balancing act of BCL-2 family proteins. Nat. Rev. Mol. Cell Biol. 20, 175-193 (2019).

28. Tonelli, C., Chio, I. I. C. \& Tuveson, D. A. Transcriptional regulation by Nrf2. Antioxid. Redox Signal. 29, 1727-1745 (2018).

29. Klotz, L. O. et al. Redox regulation of FoxO transcription factors. Redox Biol. 6, 51-72 (2015).

30. Chen, L., Belton, R. J. Jr \& Nowak, R. A. Basigin-mediated gene expression changes in mouse uterine stromal cells during implantation. Endocrinology 150, 966-976 (2009).

31. van Rensburg, H. J. J., Lai, D., Azad, T., Hao, Y. \& Yang, X. TAZ enhances mammary cell proliferation in $3 \mathrm{D}$ culture through transcriptional regulation of IRS1. Cell. Signal. 52, 12-22 (2018).

32. Gu, K. et al. TAZ promotes the proliferation and osteogenic differentiation of human periodontal ligament stem cells via the p-SMAD3. J. Cell. Biochem. 121, 1101-1113 (2020).

33. Kim, A. R. et al. TAZ stimulates liver regeneration through interleukin-6-induced hepatocyte proliferation and inhibition of cell death after liver injury. FASEB J. 33, 5914-5923 (2019).

34. Ma, L. et al. Novel insights into Dhh signaling in antler chondrocyte proliferation and differentiation: Involvement of Foxa. J. Cell. Physiol. 235, 6023-6031 (2020).

35. Das, S. K. Cell cycle regulatory control for uterine stromal cell decidualization in implantation. Reproduction 137, 889-899 (2009).

36. Xie, $\mathrm{H}$. et al. Maternal heparin-binding-EGF deficiency limits pregnancy success in mice. Proc. Natl Acad. Sci. USA 104, 18315-18320 (2007).

37. Driskill, J. H. \& Pan, D. The Hippo pathway in liver homeostasis and pathophysiology. Annu. Rev. Pathol. 16, 299-322 (2021).

38. Kajihara, T. et al. Differential expression of FOXO1 and FOXO3a confers resistance to oxidative cell death upon endometrial decidualization. Mol. Endocrinol. 20 , 2444-2455 (2006). 
39. Rausch, V. \& Hansen, C. G. The Hippo pathway, YAP/TAZ, and the plasma membrane. Trends Cell Biol. 30, 32-48 (2020).

40. Hyeon, S., Lee, H., Yang, Y. \& Jeong, W. Nrf2 deficiency induces oxidative stress and promotes RANKL-induced osteoclast differentiation. Free Radic. Biol. Med. 65, 789-799 (2013).

41. Vasquez, Y. M. et al. FOXO1 regulates uterine epithelial integrity and progesterone receptor expression critical for embryo implantation. PLoS Genet. 14, e1007787 (2018).

42. Lenaz, G. Role of mitochondria in oxidative stress and ageing. Biochim. Biophys. Acta 1366, 53-67 (1998).

\section{ACKNOWLEDGEMENTS}

This work was financially supported by the National Natural Science Foundation of China (31672503, 31873003, and 31472158), National Key Research and Development Program of China, Stem Cell and Translational Research (2017YFA0105101), and Interdisciplinary Research Funding Program for PhD Students of Jilin University (101832020DJX095).

\section{AUTHOR CONTRIBUTIONS}

B.G. and Z.P.Y. designed and directed the research; H.F.Y. and L.W.Z. performed the experiments and analyzed the data; H.F.Y., B.G., and Z.P.Y. wrote and edited the manuscript; Z.Q.Y., Y.S.W., and T.T.W. provided technical assistance. All authors read and approved the final manuscript.

\section{COMPETING INTERESTS}

The authors declare no competing interests.

\section{ADDITIONAL INFORMATION}

Supplementary information The online version contains supplementary material available at https://doi.org/10.1038/s12276-021-00655-2.

Correspondence and requests for materials should be addressed to Z.-P.Y. or B.G.

Reprints and permission information is available at http://www.nature.com/ reprints

Publisher's note Springer Nature remains neutral with regard to jurisdictional claims in published maps and institutional affiliations.

(C)

Open Access This article is licensed under a Creative Commons Attribution 4.0 International License, which permits use, sharing, adaptation, distribution and reproduction in any medium or format, as long as you give appropriate credit to the original author(s) and the source, provide a link to the Creative Commons license, and indicate if changes were made. The images or other third party material in this article are included in the article's Creative Commons license, unless indicated otherwise in a credit line to the material. If material is not included in the article's Creative Commons license and your intended use is not permitted by statutory regulation or exceeds the permitted use, you will need to obtain permission directly from the copyright holder. To view a copy of this license, visit http://creativecommons. org/licenses/by/4.0/

(c) The Author(s) 2021 